\title{
Pathophysiology of diabetic retinopathy
}

\author{
J. G. CUNHA-VAZ \\ From the Department of Ophthalmology, University of Coimbra, Coimbra, Portugal
}

SUMmARY After a brief analysis of the pathological picture of diabetic retinopathy, of which only the topographical distribution of the vascular lesions appears to be specific, the results obtained with 2 new methods of study of the retinal circulation, are presented. These methods are vitreous fluorophotometry and fluorometric determination of segmental retinal blood flow. Vitreous fluorophotometry has shown that a disturbance of the blood-retinal barrier, possibly functional, appears in diabetic eyes before any lesion is clinically visible in the fundus, and that there is a close correlation between the severity of the vascular lesions and higher vitreous fluorophotometry readings. Blood flow studies have shown that in diabetes the retinal blood flow increases markedly with progress of background retinopathy, decreasing finally where proliferative retinopathy, with marked arteriolar narrowing, is present.

On the basis of these findings a working hypothesis for the pathogenesis of diabetic retinopathy is presented.

Diabetic retinopathy is one of the most challenging problems facing ophthalmological research. Its incidence continues to increase all over the world, so that today it is one of the most frequent causes of blindness. The incidence of blind registrations due to diabetes is now more than 15 million a year. This situation is particularly serious because, although much is known about the pathology of diabetic retinal disease, practically nothing is known about its cause.

Ashton, who has contributed so extensively to our knowledge of diabetic retinopathy, remarked in 1974 that "we must continue to look for more fundamental scientific investigations and at the same time develop new ways of examining the diabetic retina in an effort to unravel the still unsolved mysteries of diabetic retinopathy'.

In this presentation an effort will be made to review the present state of knowledge on the pathophysiology of diabetic retinopathy, with particular emphasis on personal studies using new methods of examining the retinal circulation and blood-retinal barrier.

\section{Pathology}

Diabetic retinopathy is basically a microangiopathy, the usual, initial presenting sign of which is the

Address for reprints: Professor José Cunha-Vaz, Clinica Oftalmológica, Hospitais da Universidade de Coimbra, Celas, Coimbra, Portugal appearance of retinal microaneurysms at the posterior pole. In 1972 I examined the pathology of diabetic retinopathy and other vascular retinopathies using injection methods, a variety of stains, and the digestion technique. Light microscopic examination of retinal 'digests' is particularly appropriate to study alterations in the retinal vascular bed. From this study a clear pattern of disease was observed to occur in diabetic retinopathy. The results suggested that changes are confined to the small vessels in the form of endothelial proliferation, rare microaneurysms, and signs of impending cellular degeneration in a few vascular branches. These initial lesions are focal and located preferentially at the posterior pole of the retina.

At first the endothelial proliferation and microaneurysms appear to be confined to the venous side of the retinal circulation, whereas at this stage endothelial degeneration changes appear to be limited to capillaries on the arterial side of the circulation (Fig. 1).

The pericytes, although showing clear signs of disease, are affected in a highly irregular manner.

With progression of the disease the capillaries on the arterial side of the retinal circulation show increased cell loss and closure. Simultaneously, on the venous side of the circulation, there is an increase in the number of microaneurysms. As the areas of capillary closure enlarge, they are seen to be traversed by a few enlarged capillaries, which appear to act as arteriovenous shunts, receiving the 
blood diverted from the surrounding closed capillary net. These observations are summarised in Table 1.

Are these lesions specific for diabetes? Examination of other vascular retinopathies emphasises the probable importance of local factors and that the lesions described in diabetic retinopathy are shared by a wide variety of apparently unrelated diseases.

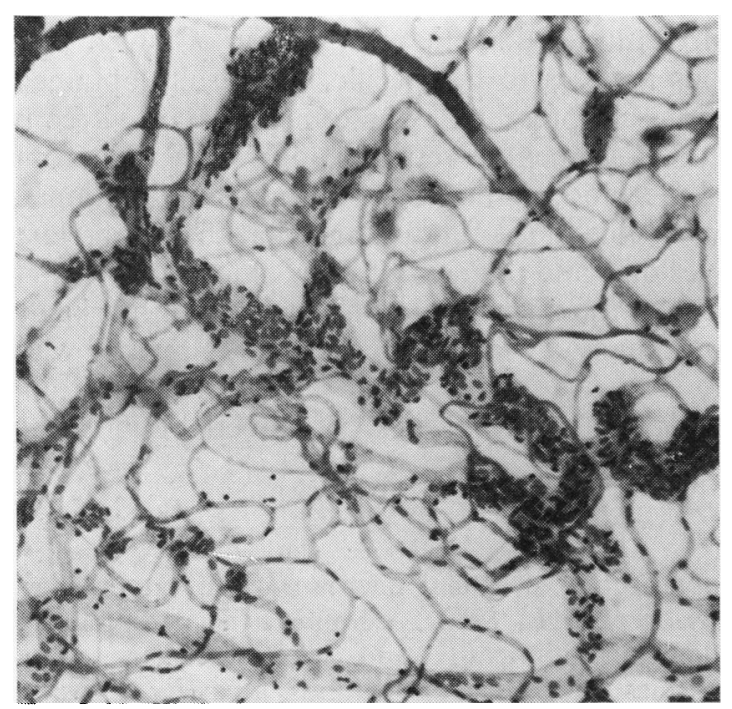

Fig. 1 Diabetic retinopathy, showing capillary closure in the arterial side and the presence of endothelial proliferation and microaneurysms in the venous side. Digest preparation. (PAS- $\mathrm{H} \times 85$ )
Endothelial proliferation, prevailing on the venous side of the circulation, is a frequent finding in polycythaemia, leukaemia, myelomatosis, circinate retinopathy, central retinal vein thrombosis, macroglobulinaemia and Eales's disease.

Microaneurysms are probably the commonest retinal lesion in diabetes (Ashton, 1963) but they are also seen, frequently in large numbers, in circinate retinopathy, Eales's disease, leukaemia, myelomatosis, macroglobulinaemia, central retinal vein thrombosis, hypertensive retinopathy, glaucoma, and retinoblastoma.

Areas of capillary closure are seen in scleroderma, hypertensive retinopathy, pernicious anaemia, and central retinal vein thrombosis (Fig. 2).

Arteriovenous shunts appear in circinate retinopathy and central retinal vein thrombosis. Basement membrane thickening and pericyte degeneration are similarly found in other retinopathies, but because of their especial prevalence in diabetic retinopathy they merit further consideration.

\section{BASEMENT MEMBRANE THICKENING}

This is believed to play an important role not only in diabetic retinopathy but in diabetic microangiopathy as a whole. Ashton reviewed the subject in detail in 1974, commenting on basement membrane thickening in several non-diabetic conditions, its inconstancy, and its presence in all types of diabetes. Electron microscopical studies of diabetic retinopathy show that basement membrane thickening is associated with a variety of haematogeneous elements, which could have arrived at this situation

Table 1 Evolution of retinal vascular lesions in diabetes

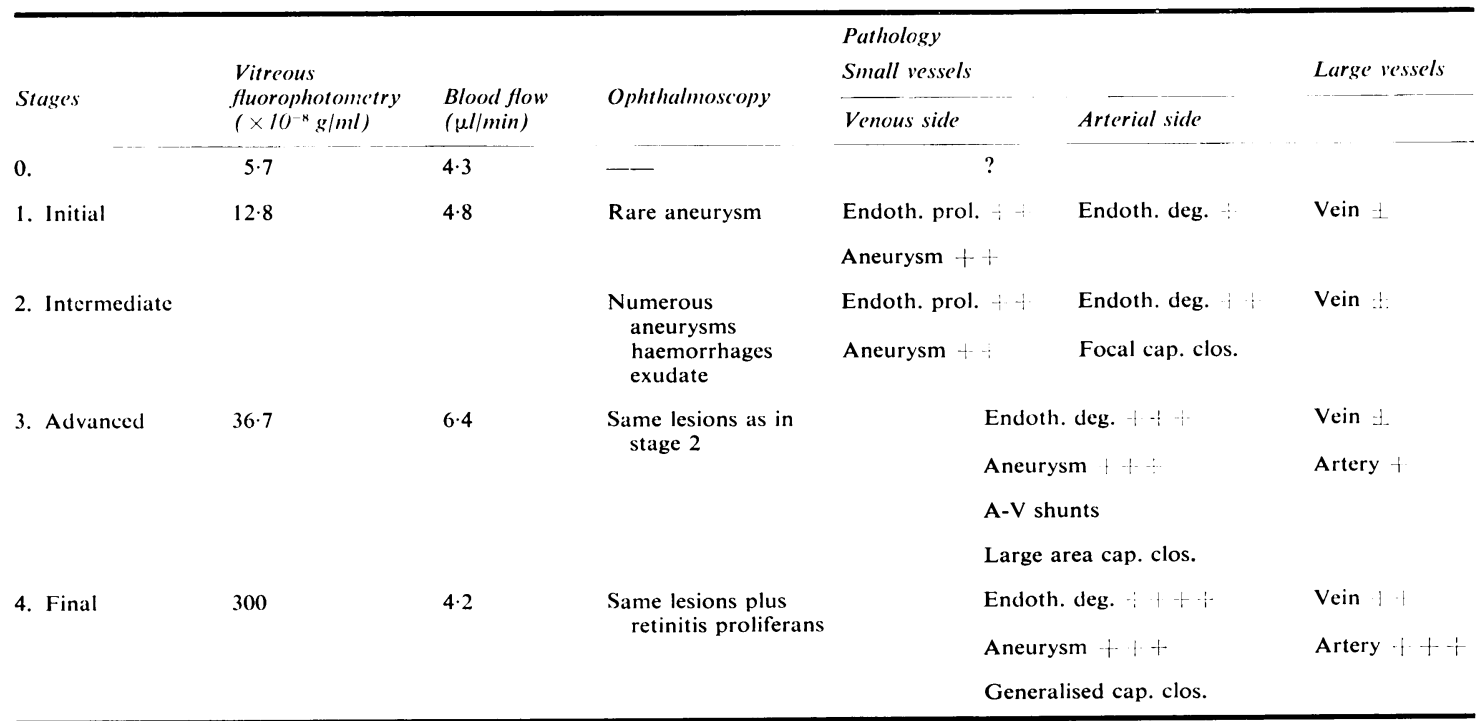




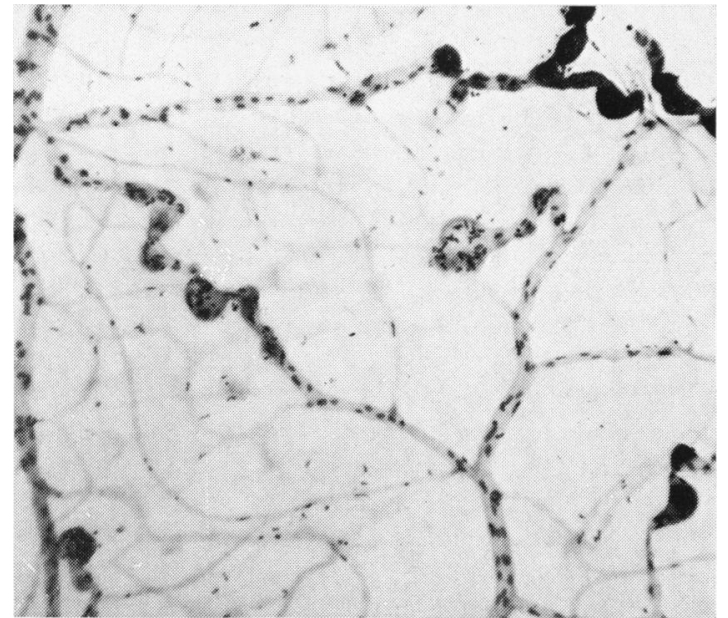

Fig. 2 Central retinal vein thrombosis, showing a marked degree of capillary closure. The capillary net has been replaced by a few dilated anastomotic vessels presenting endothelial proliferation and microaneurysms. Digest preparation. $(\mathrm{PAS}-\mathrm{H} \times 77$ )

only through leakage due to breakdown of the endothelial barrier. Leakage of plasma components is especially evident in microaneurysms and is apparently responsible for the greatly thickened wall. It is also important to recall that leakage of this kind necessarily implies a preceding endothelial injury.

In his electron micrographs of diabetic basement membranes Ashton observed a characteristic multilayered appearance with interposed cellular debris, a finding which Vracko and Benditt (1970) believe to be due to repeated endothelial cell death with regeneration and replacement, each new generation of cells contributing their own basement laminae and entrapping the cellular debris of the dead cells. This may explain the comparatively healthy appearance of the endothelium even in the presence of gross periendothelial pathology, including degenerate pericytes. Basement membrane thickening appears, therefore, to be a secondary feature of the disease, due in part, possibly, to glycoprotein insudation from an initial endothelial dysfunction, as considered by Williamson and Kilo (1976).

\section{PERICYTE DAMAGE}

There is abundant evidence that these cells are selectively involved in the diabetic process (Cogan and Kuwabara, 1963), the damage taking the form of a peculiar eosinophilic degeneration of the nucleus before the cell disintegrates altogether. Although similar changes can be observed in other retinopathies, they are by no means so common as in diabetes.
DISTRIBUTION OF RETINAL VASCULAR LESIONS It has been shown that there is no one lesion which is absolutely specific for diabetic retinopathy. The most characteristic features of diabetic retinopathy, microaneurysms, capillary closure, basement membrane thickening, and pericyte damage appear to differ from other vascular retinopathies only in their frequency and widespread distribution. While the vascular involvement in other retinopathies is localised initially either to the arterial or to the venous side of the circulation (Cunha-Vaz, 1967; Wise et al., 1971), there are indications that in diabetic retinopathy the entire vascular tree at the posterior pole of the retina is involved from the beginning.

\section{Recent clinical research}

The advent of fluorescein angiography and photocoagulation has had an enormous impact on the study of diabetic retinopathy, the former teaching us much about the natural history of retinopathy although without giving much new information about the underlying defect (Ashton, 1976).

Recently, however, two new clinical methods of study of the retinal circulation, vitreous fluorophotometry and fluorometric determination of segmental retinal blood flow, have been developed in our department. These methods have given valuable information on the physiopathogenesis of diabetic retinopathy.

Vitreous fluorophotometry is a clinical method for the quantitative study of the blood-retinal barrier (Cunha-Vaz et al., 1975). The examination of a number of diabetic patients presenting different degrees of retinopathy showed a significant breakdown of the blood-retinal barrier in every eye examined. Furthermore, it was found that disturbance of the blood-retinal barrier, as shown by vitreous fluorophotometry, appears before any lesions such as capillary closure and microaneurysms are clinically visible in the fundus. This study showed that a breakdown of the blood-retinal barrier, possibly functional, is undoubtedly one of the earliest changes to occur in the retina in diabetes. Using vitreous fluorophotometry we have now examined 45 patients with confirmed diabetes, but an apparently normal retina as shown by ophthalmoscopy and fundus fluorescein angiography and having 10/10 vision. In spite of the 'normal' fluorescein angiograms, the vitreous fluorophotometry readings were constantly above normal, with average values of $5.7 \times 10^{-8} \mathrm{~g} / \mathrm{ml}$ of fluorescein in the posterior vitreous and $4.7 \times 10^{-8} \mathrm{~g} / \mathrm{ml}$ in the middle vitreous 1 hour after an intravenous injection of $1 \mathrm{~g}$ of sodium fluorescein (Table 2).

Another point that emerged from our studies 
and which deserves further attention is the probable role of alteration of the blood-retinal barrier in the progress of diabetic retinopathy, as testified by the close correlation between the severity of the vascular lesions and the vitreous fluorophotometry readings. Vitreous fluorophotometry of 24 diabetic patients presenting simple background retinopathy characterised by moderate numbers of microaneurysms but no signs of macular involvement (Fig. 3), showed average values of $12 \cdot 8 \times 10^{-8} \mathrm{~g} / \mathrm{ml}$ of fluorescein in the posterior vitreous and $10.7 \times 10^{-8} \mathrm{~g} / \mathrm{ml}$ in the middle vitreous, whereas average values of $36.7 \times 10^{-8} \mathrm{~g} / \mathrm{ml}$ of fluorescein were recorded in the posterior vitreous of diabetic patients showing numerous microaneurysms, haemorrhages, dilated vascular channels, and areas of capillary closure with signs of macular involvement, including coalescing hard exudates (Fig. 4).

As regards the pathophysiology of diabetic retinopathy, vitreous fluorophotometry has, therefore, contributed by demonstrating that a breakdown of the blood-retinal barrier is apparently the earliest change to occur in the retinae of diabetic patients. This observation has since been confirmed by electron microscopy in alloxan-diabetic dogs and shown to be associated with opening of the interendothelial junctions (Wallow and Engerman, 1977). Similarly, the fact that a close correlation between progression of the vascular disease and increasing vitreous fluorophotometry readings was observed indicates that the altered permeability of the blood-retinal barrier is likely to play an important role in the development of the entire pathological picture of diabetic retinopathy.

The development of a new direct method for estimation of human retinal blood flow by slit-lamp fluorophotometry has also proved useful (CunhaVaz and Lima, 1977). Examination of a group of diabetic patients showed close correlation between increased retinal blood flow and the clinical staging of the retinopathy (Table 1). Retinal blood flow is within normal limits in the initial stages of retinal involvement in diabetes, when the only alteration is a breakdown of the blood-retinal barrier.

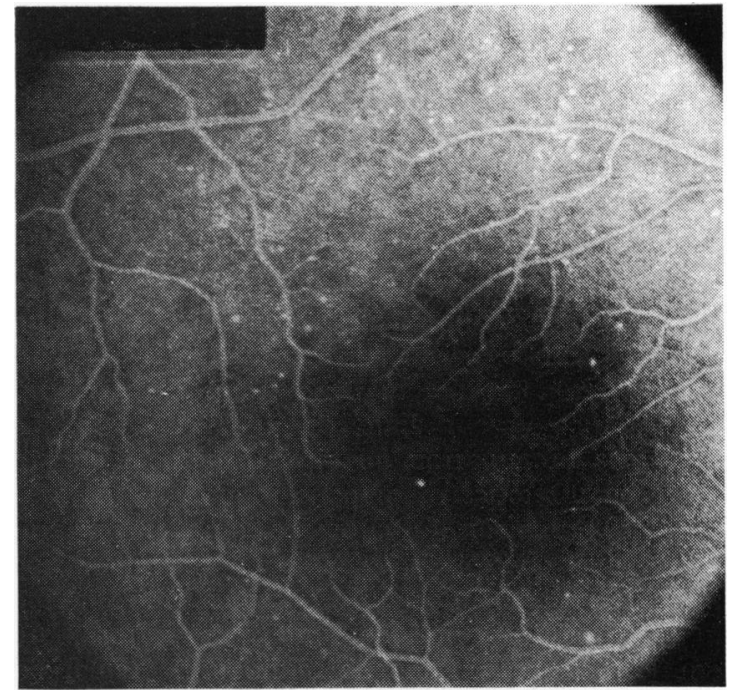

Fig. 3 Fluroescein angiogram of a case classified as background retinopathy without maculopathy

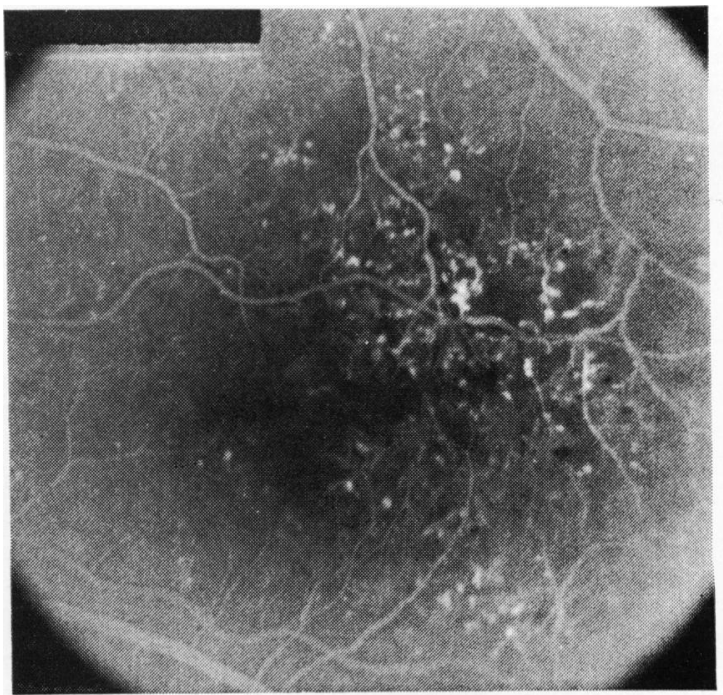

Fig. 4 Fluorescein angiogram of a case classified as background retinopathy with maculopathy

Table 2 Vitreous fluorophotometry and superior temporal blood flow in diabetic patients

\begin{tabular}{|c|c|c|c|c|c|}
\hline \multirow{2}{*}{ Retinal involvement in diabetes } & \multicolumn{2}{|c|}{$\begin{array}{l}\text { Vitreous fluorophotometry } \\
\left(\times 10^{-8} \mathrm{~g} / \mathrm{ml}\right)\end{array}$} & \multicolumn{3}{|c|}{$\begin{array}{l}\text { Segmental blood flow } \\
\text { (Sup. temporal) }\end{array}$} \\
\hline & Posterior & Middle & $\begin{array}{l}\text { Blood flow } \\
\text { (litre/min) }\end{array}$ & $\begin{array}{l}\text { Transit time } \\
\text { (second) }\end{array}$ & $\begin{array}{l}\text { Arterial } \\
\text { diameter }(\mu \mathrm{m})\end{array}$ \\
\hline 'Normal retina' & $5 \cdot 7 \pm 1 \cdot 9$ & $4 \cdot 7 \pm 1 \cdot 7$ & $4 \cdot 3 \pm 0 \cdot 5$ & $0 \cdot 36 \pm 0.05$ & $188 \pm 15$ \\
\hline Simple background retinopathy & $12 \cdot 8 \pm 6 \cdot 3$ & $10 \cdot 7 \pm 4 \cdot 9$ & $4 \cdot 8 \pm 0 \cdot 6$ & $0.29 \pm 0.05$ & $179 \pm 18$ \\
\hline Background retinopathy with maculopathy & $36 \cdot 7 \pm 14 \cdot 2$ & $28 \cdot 5 \pm 11 \cdot 1$ & $6 \cdot 4 \pm 1 \cdot 1$ & $0.23 \pm 0.06$ & $183 \pm 18$ \\
\hline
\end{tabular}


Patients with minimal background retinopathy show a slight increase in retinal blood flow, whereas marked increase of segmental blood flow is observed in the more advanced stages of background retinopathy with maculopathy.

Patients presenting with proliferative retinopathy, however, have lower near-normal retinal blood flow values. The results obtained in late-onset diabetic proliferative retinopathy point clearly to the 'occlusive' nature of the retinopathy.

A number of matters relevant to the pathophysiology of diabetic retinopathy are highlighted by this study. In the first place it appears that changes in retinal blood flow are not the earliest finding in diabetes, and the question arises as to why progression of the disease should give rise to increased flow. Probably capillary and venous dilatation is responsible for the initial increase in blood flow in simple background retinopathy. Later on, endothelial damage and progressive thickening of the basement membrane lead to progressive capillary closure and the development of arteriovenous shunts which short-circuit the capillary bed and shorten its length. This factor, associated with progressive loss of autoregulation on the part of the retinal arteries, is the most probable cause of increased blood flow in advanced background retinopathy.

Also to be considered is the possibility that the observed increase in retinal blood flow may itself be deleterious to the retina and responsible for further advancement of the disease. In this context it may be relevant to note that conditions which are associated with decreased blood flow, for example, carotid insufficiency, high myopia, and optic nerve atrophy, have a beneficial effect on the evolution of diabetic retinopathy.

The recent clinical studies outlined above provide the basis for a working hypothesis of the evolution of diabetic retinopathy. It appears to start at the level of the endothelial membrane of the small retinal vessels with reversible breakdown of the inner blood-retinal barrier. The initial changes in the capillary cell population, which include endothelial proliferation on the venous side and generalised pericyte damage, are possibly related to the capillary-venous dilatation linked with the initial increase in blood flow. Basement membrane thickening associated with endothelial swelling or proliferation might be responsible for the capillary closure and subsequent shunt formation. Shunt formation and loss of arteriolar autoregulation appear to be involved in the increase of retinal blood flow at this later stage.

While local factors appear to be responsible for the entire evolution, once the process has been started by changes in the blood-retinal barrier, the absence of any clear relation between diabetic control and progression or regression of the retinopathy suggests that subsequent development is largely autonomous. There is, however, evidence of a direct relationship between the initial breakdown of the blood-retinal barrier and diabetic control (Cunha-Vaz et al., 1977).

I think that there is good support for the view that processes of two kinds take part in the pathogenesis of diabetic retinopathy. There is, in the first place, a generalised disorder of small blood vessels of unknown cause which is specific to diabetes, metabolically determined, and at least in part related to the degree and duration of departure from metabolic normality. And in the second place there are local responses specific to the retina, conditioned by its vascular peculiarities and the patient's age, which, once initiated, are largely selfperpetuating.

This study was supported by Centro de Oftalmologia do Instituto Nacional de Investigação Científica, Portugal.

\section{References}

Ashton, N. (1963). Studies of the retinal capillaries in relation to diabetic and other retinopathies. British Journal of Ophthalmology, 47, 521-538.

Ashton, N. (1974). Vascular basement membrane changes in diabetic retinopathy. British Journal of Ophthalmology, 58, 344-366.

Ashton, N. (1976). Pathology of diabetic retinopathy. In Acta XXII Concilium Ophthalmologicum, Vol. 1, pp. 175-181. Masson: Paris.

Cogan, D. G., and Kuwabara, T. (1963). Capillary shunts in the pathogenesis of diabetic retinopathy. Diabetes, 12 , 293-300.

Cunha-Vaz, J. G. (1967). In Aspectos da Fisiopatologia Vacular da Retina. Imprensa de Coimbra: Coimbra.

Cunha-Vaz, J. G. (1972). Diabetic retinopathy: human and experimental studies. Transactions of the Ophthalmological Societies of the United Kingdom, 92, 111-124.

Cunha-Vaz, J. G., Abreu, J. R., Campos, A. J., and Figo, G. (1975). Early breakdown of the blood-retinal barrier in diabetes. British Journal of Ophthalmology, 59, 649-656.

Cunha-Vaz, J. G., Fonseca, J. R., Abreu, J. R., and Ruas, M. (1977). (Unpublished observations.)

Cunha-Vaz, J. G., and Lima, J. P. (1977). Archives of Ophthalmology. 00, 000-000.

Vracko, R., and Benditt, E. P. (1970). Capillary basal lamina thickening: its relationship to endothelial cell death and replacement. Journal of Cell Biology, 47, 281.

Wallow, I. H., and Engerman, R. L. (1977). Permeability and patency of retinal blood vessels in experimental diabetes. Investigative Ophthalmology and Visual Science, 16, 447-461.

Williamson, J. R., and Kilo, C. (1976). Basement-membrane thickening and diabetic microangiopathy. Diabetes, 25, 925-927.

Wise, G. N., Dollery, C. T., and Henkind, P. (1971). The Retinal Circulation. Harper \& Row: New York. 